\title{
Treatment of primary full-thickness macular hole by intravitreal injection of expansile gas
}

\author{
Ruoan Han ${ }^{1} \cdot$ Chenxi Zhang ${ }^{1} \cdot$ Xinyu Zhao ${ }^{1} \cdot$ Youxin Chen ${ }^{1}$ \\ Received: 31 December 2016 / Revised: 6 February 2018 / Accepted: 16 February 2018 / Published online: 5 September 2018 \\ (c) The Royal College of Ophthalmologists 2018
}

\begin{abstract}
Objective To evaluate the efficacy of intravitreal injection (IVI) of expansile gas to treat primary full-thickness macular hole (FTMH).

Methods Prospective interventional case series. Twenty-six eyes of 26 patients in Peking Union Medical College Hospital with primary FTMH underwent IVI of perfluoropropane $\left(\mathrm{C}_{3} \mathrm{~F}_{8}\right) 0.2 \mathrm{ml}$, followed by $7-14$ days' face-down positioning. The macular hole closure rate, post-operative visual acuity, and the incidence of surgical complications were observed. The correlation between the patients' age, macular hole diameter, pre-operative best-corrected visual acuity (BCVA), status of the vitreous, and the macular hole closure rates were analyzed.

Results A total of 17 cases (65.4\%) had hole closure after IVI of $\mathrm{C}_{3} \mathrm{~F}_{8}$ alone. The closure rate of small, medium, and large FTMH was $85.7,80$, and $33.3 \%$, respectively. Pre-operative OCT examination showed that 10 cases $(37 \%)$ had vitreomacular traction (VMT), and all of them achieved vitreous-macula separation after IVI of the gas. The diameters of the hole $(P=0.024)$ and the status of the vitreous $(P=0.038)$ had a significance difference for hole closure. The mean pre-operative and post-operative BCVA was $0.84 \pm 0.29$ and $0.49 \pm 0.36(\operatorname{logMAR})$, respectively $(P<0.01)$. One myope with persistent hole developed rhegmatogenous retinal detachment (RRD) 2 months after the surgery. Another myope with hole closure developed RRD 10 months after the surgery. They achieved hole closure and retinal reattachment after vitrectomy and scleral buckling, respectively.

Conclusion Intravitreal injection of $\mathrm{C}_{3} \mathrm{~F}_{8}$ gas alone was an effective treatment for small-sized and medium-sized primary FTMH, but further large prospective studies are needed.
\end{abstract}

\section{Introduction}

Macular hole is a common macular disease that causes significant impairment of the central vision. Primary fullthickness macular hole (FTMH) results from vitreous traction on the fovea from abnormal posterior vitreous detachment (PVD). The Gass classification was based on clinical examination and divided the macular holes into four stages, with stage 1 representing impending hole and stages 2-4 representing FTMHs [1, 2]. In 2013, the International Vitreomacular Traction Study (IVTS) Group developed an optical coherence tomography (OCT)-based anatomic

Youxin Chen

chenyouxinpumch@163.com

1 Department of Ophthalmology, Peking Union Medical College Hospital, No.1 Shuai fu yuan, Dongcheng District, 100730 Beijing, China classification system for diseases of the vitreomacular interface (VMI) [3]. According to the classification system, FTMH is defined as a foveal lesion, with interruption of all retinal layers from the internal limiting membrane (ILM) to the retinal pigment epithelium (RPE). Primary FTMH is caused by vitreous traction. FTMH is subclassified by the size of the hole, as determined by OCT and the presence or absence of vitreomacular traction (VMT). A small FTMH is defined by a diameter of less than $250 \mu \mathrm{m}$, and a medium FTMH features aperture size from 250 to $400 \mu \mathrm{m}$, while a large FTMH with an aperture of larger than $400 \mu \mathrm{m}$. Pars plana vitrectomy (PPV), gas-fluid exchange, and face-down positioning with or without ILM peeling is the first treatment procedure for medium and large FTMH.

Vitrectomy to FTMH brings out significant benefits, but it has some potential limitations. Reported complications include cataract formation, RPE alteration, retinal detachment (RD), cystoid macular edema (CME), visual field defects, choroidal neovascularization, and 
endophthalmitis $[4,5]$. The potential adverse events support searching for less invasive and simple methods to treat FTMH.

In previous studies, intravitreal injections (IVI) of expansile gas was a useful way to treat early idiopathic macular holes, but Chen et al. showed a low success rate [69]. In this study, we examined patients with primary FTMH and evaluated whether the morphological and functional outcomes of gas injection only were of potential benefit as the first line of therapy.

\section{Materials and methods}

\section{Patients and study design}

This is a prospective, consecutive, interventional case series. Consecutive patients with diagnosis of primary FTMH, but without other ocular complications, were enrolled in this study. Excluded were cases with secondary macular holes, as for FTMH associated with blunt trauma, lightning strike, pathologic myopia, macular schisis, and retinal vascular diseases. Patients having peripheral retinal holes or lattice degeneration were also excluded. Twenty-six eyes (12 right and 14 left) of 26 patients with primary FTMH were enrolled in this study. The patient population consisted of 21 women $(80.8 \%)$ and 5 men (19.2\%) with age ranging from 25 to 77 years $(59 \pm 12$ [mean \pm standard deviation]). The follow-up period was 6 months after surgery. Pre-treatment logarithm of the minimum angle of resolution (logMAR) bestcorrected visual acuity (BCVA) was within 0.3 to 1.5 . The average pre-treatment $\log$ MAR BCVA was $0.85 \pm 0.29$. Diagnosis and classification of macular holes were based on slit-lamp biomicroscopic and OCT examinations using the IVTS classification system. The study was approved by the Institutional Ethics Committee of the Chinese Academy of Medical Sciences, Peking Union Medical College Hospital. Study procedures conformed to the tenets of the Declaration of Helsinki. Written informed consent was obtained from all participants.

\section{Pre-treatment and post-treatment examinations}

All patients underwent comprehensive ocular evaluation before treatment, including BCVA testing, slit-lamp biomicroscopic examination, indirect ophthalmoscopy, fundus photography, and OCT examination. The baseline data included identification of the study eye, BCVA, diameter measured by OCT, classification of the macular hole, description of the vitreous status. Hole closure was defined as perfect apposition of the edges that was determined by biomicroscopy and OCT examinations. BCVA was examined by standard logarithmic visual acuity chart and converted to logMAR VA for measuring VA changes and for statistical analysis.

OCT was taken through a dilated pupil by a trained examiner with knowledge of clinical findings of the retinal disease using two different available systems (3D OCT2000 Spectral Domain, Topcon, Japan; Spectralis, Heidelberg Engineering, Germany). Standardized horizontal and vertical vitreoretinal sections through the macular hole were routinely collected. Diameters of the macular holes were measured in horizontal scans by one doctor using the OCT caliper function, at the narrowest hole point in the mid retina, as a line drawn roughly parallel to the RPE.

\section{Surgical procedure}

Under topical anesthesia, proper disinfection, and placing lid speculum, anterior chamber paracentesis was followed by IVI of $100 \%$ perfluoropropane $\left(\mathrm{C}_{3} \mathrm{~F}_{8}\right)$ of $0.2 \mathrm{ml}$ through the pars plana using a 30 -gauge needle and a 1-ml syringe. All operations were performed by the same surgeon (CYX). Patients were kept in prone position while awake for 7 days after treatment. If the hole remained open, further head-down position was asked, for a total of 14 days. Visual acuity, intraocular pressure, slit-lamp biomicroscopic examination, and indirect ophthalmoscopy were performed 1 day, 1 week, and 1 month after the procedure. From 1 month, OCT was also performed to observe the healing of the macular hole. The patient was scheduled for vitreous surgery if the hole remained unsealed or certain complications developed. The surgical procedure included 3-port PPV, posterior hyaloid removal, ILM peeling, and gas/air tamponade.

\section{Statistical analysis}

Data analysis was carried out using SPSS 19.0 software. Continuous values in text were expressed as mean \pm standard deviation. Categorical variables were reported as percentages. A paired or unpaired $t$-test was used to compare the baseline characteristics and the treatment outcomes before and after the treatment. The significance level for all statistical tests was set to $P<0.05$.

\section{Results}

\section{Post-treatment macular hole and VMI status}

A total of 26 eyes of 26 patients with primary FTMH were enrolled in this study, within them, 7 eyes were small FTMH, 10 eyes were medium FTMH, and 9 eyes were large FTMH. VMT was delineated by OCT in ten eyes $(37 \%)$, and all of them $(100 \%)$ were released after the 


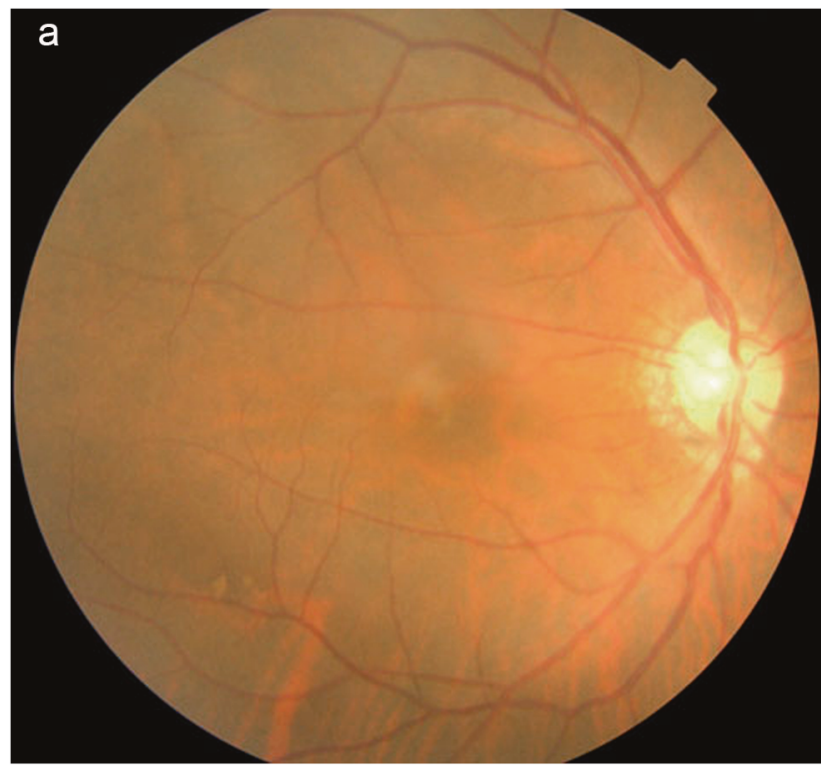

Fig. 1 Fundus photograph (a) and optical coherence tomography (OCT) (b) images of the medium full-thickness macular hole (FTMH) in right eye with a diameter of $399 \mu \mathrm{m}$, with vitreomacular traction (VMT). After 1 month (c) of intravitreal injection of expansile gas, the

injection of the gas. Within the ten eyes, hole closure rate was $90 \%$ (9 of 10). In 17 cases (65.4\%), IVI of expansile gas alone resulted in anatomical closure of the macular hole (Figs. 1, 2). Hole closure was achieved in 6 of the 7 eyes (85.7\%), when the hole diameter was smaller than $250 \mu \mathrm{m}$ (small FTMH), while closure number of medium and large FTMH was 8 of 10 eyes (80\%) and 3 of 9 eyes (33.3\%), respectively. All nine cases in which the anatomical success was not achieved by intraocular gas injection alone then underwent vitreous surgery within 1 month. All nine of these macular holes were closed successfully with a single surgical procedure (Fig. 3). The clinical features were shown in Table 1.

\section{Relationships between baseline factors and anatomical holes closure after intravitreal gas injection alone}

Baseline factors including age, gender, hole diameter, pretreatment BCVA, and VMT status were analyzed for the association with anatomical hole closure after IVI alone. There was a significant difference in hole diameter between eyes that were successfully closed and eyes that did not close after the procedure $(P=0.024 ; 307.8 \pm 122.8 \mu \mathrm{m}$, $431.6 \pm 128.4 \mu \mathrm{m}$, respectively). The presence of VMT was a factor significantly related to hole closure $(P=0.038)$. Age $(P=0.052)$, gender $(P=0.465)$, and initial BCVA $(P$ $=0.262)$ did not show statistically significant association with macular hole closure.

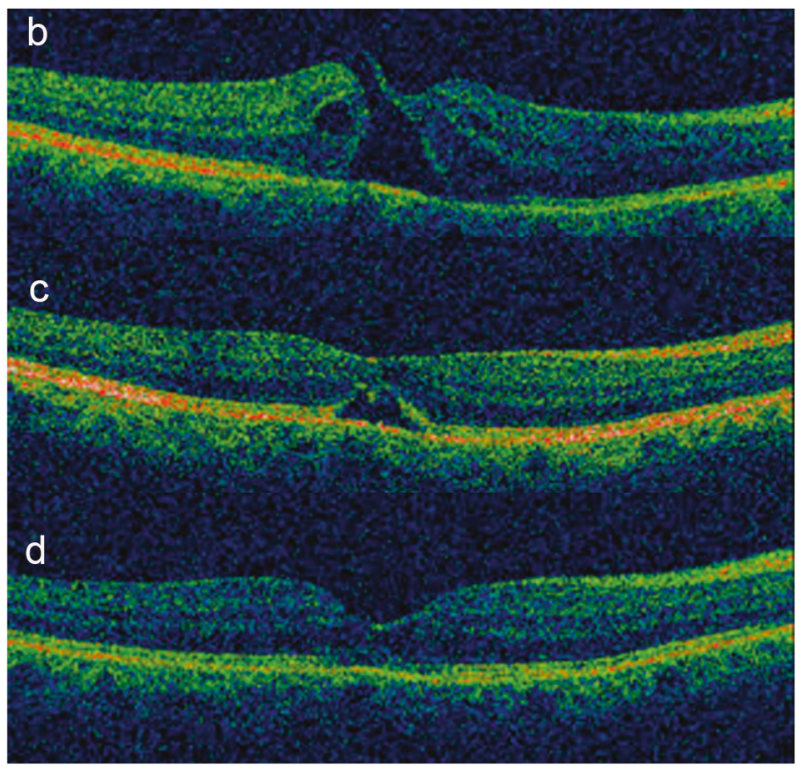

vitreous detachment and the anatomical closure of the hole was observed. After 2 months (d) of the surgery, a hole closure was completed and the macular fovea was almost normal. Corrected visual acuity was improved from 0.1 (pre-treatment) to 0.4 (post-treatment)

\section{Post-treatment BCVA and influence factors}

In 26 eyes, 20 eyes $(76.9 \%)$ achieved better BCVA after gas tamponade alone, 2 eyes (7.7\%) showed the same BCVA, and 4 eyes (15.4\%) attained slightly decreased BCVA. $\log$ MAR vision improved from $0.84 \pm 0.29$ to $0.49 \pm 0.36$ in all patients $(t=4.887, P<0.01)$. The mean pre-treatment and post-treatment $\operatorname{logMAR}$ BCVA and $P$-values of different groups were showed in Table 2 .

\section{Adverse events}

In nine eyes without macular hole closure after gas injection alone, one case with a hole diameter of $431 \mu \mathrm{m}$ and -5.0D myopia developed localized macular-on RD 2 months after pneumatic vitreolysis. PPV and ILM peeling was done shortly after the diagnosis. After the surgery, the macular hole was closed and the RD was repaired, but BCVA remained the same. In 17 eyes with macular hole closure, one case with hole diameter of $384 \mu \mathrm{m}$ and $-4.0 \mathrm{D}$ myopia developed localized macularon RD 10 months after the surgery. After scleral buckling, RD was successfully repaired and the patient had improvement in visual acuity. There was no reopening of the hole, CME, epiretinal membrane, choroidal neovascularization, endophthalmitis, or vitreous hemorrhage. 

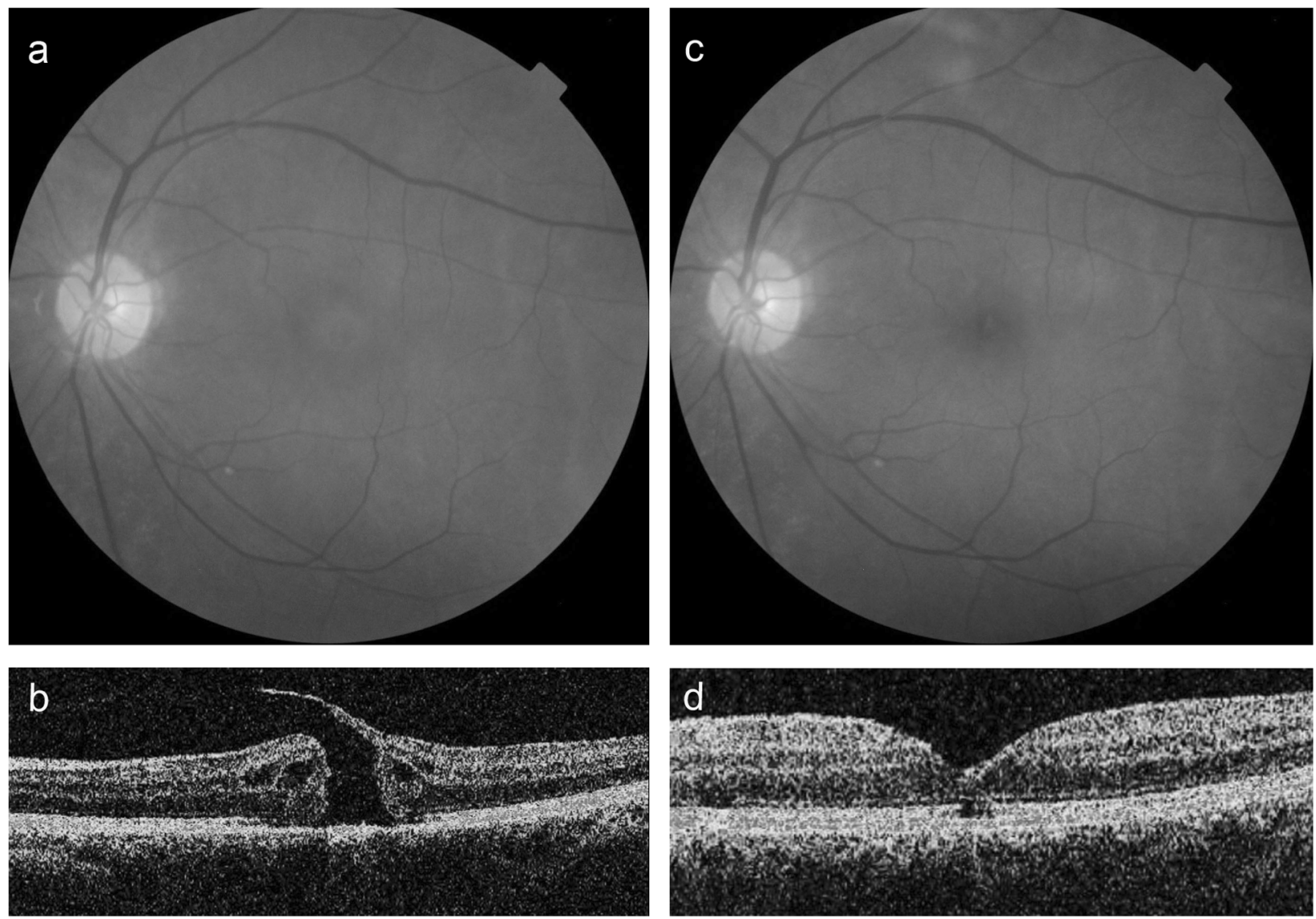

Fig. 2 Fundus photograph (a) and optical coherence tomography (OCT) (b) images of the large full-thickness macular hole (FTMH) in the left eye with a diameter of $454 \mu \mathrm{m}$, with vitreomacular traction (VMT). After 1 month $(\mathbf{c}, \mathbf{d})$ of intravitreal injection of expansile gas,

the vitreous detachment and the anatomical closure of the hole was observed. Corrected visual acuity was improved from 0.15 (pretreatment) to 0.3 (post-treatment)

\section{Discussion}

Gas tamponade to treat idiopathic macular hole had been reported in several case series [6-9]. Chan et al. [6] found that gas bubble can accelerate posterior cortical vitreous detachment by relieving VMT and cause high success closure rate for stage 1 holes and 50\% closure rate for stage 2 holes. Jorge et al. [7] reported a 50\% closure rate for stage 2 holes. Mori et al. [8] treated 20 cases of stage 2 holes and had a $50 \%$ aret of hole closure. They also found that successful closure of macular holes occurs with significantly higher frequency in smaller holes and holes associated with better pre-treatment vision. However, Chen et al. [9] treated 12 cases of stage 2 holes and only had a $25 \%$ rate of hole closure. In our series, we provide evidence that an IVI of an expansile concentration (100\%) of perfluoropropane $\left(\mathrm{C}_{3} \mathrm{~F}_{8}\right)$ gas $(0.2 \mathrm{ml})$ will induce detachment of the posterior vitreous and cause anatomical closure rate of $65.4 \%$. The use of intravitreous expansile gas alone does not appear to affect the likelihood of subsequent success with conventional macular hole surgery, and the average BCVA elevates significantly.

We found that the closure rate of small FTMHs is $85.7 \%$, and closure rates of medium and large FTMHs are 80 and $33.3 \%$, respectively, after intravitreous injection of expansile gas alone. Smaller holes had higher successful closure rate. According to the previous studies, small FTMHs got a high closure rate with vitrectomy (approaching 100\%) [3], and post-surgical medium FTMHs closure rates were also very high $(>90 \%)[10,11]$. Even for large holes, vitrectomy with ILM peeling was associated with high closure rate $(90-95 \%)$. Without an ILM peeling, the vitrectomy success rate was closer to $75 \%$ [12]. According to our study, IVI of expansile gas alone had a closure rate of over $80 \%$ for small and medium FTMHs. The closure rate by intravitreal expansile gas is lower than vitrecotomy, but this procedure has its own advantages such as limited invasiveness and procedure time, cost-saving, ease of the surgery procedure and anesthesia, and lower rate of severe complications.

Most of the patients (76.9\%) in our study achieved an improvement in BCVA after primary gas tamponade. Mean BCVA significantly improved, compared with baseline. It 

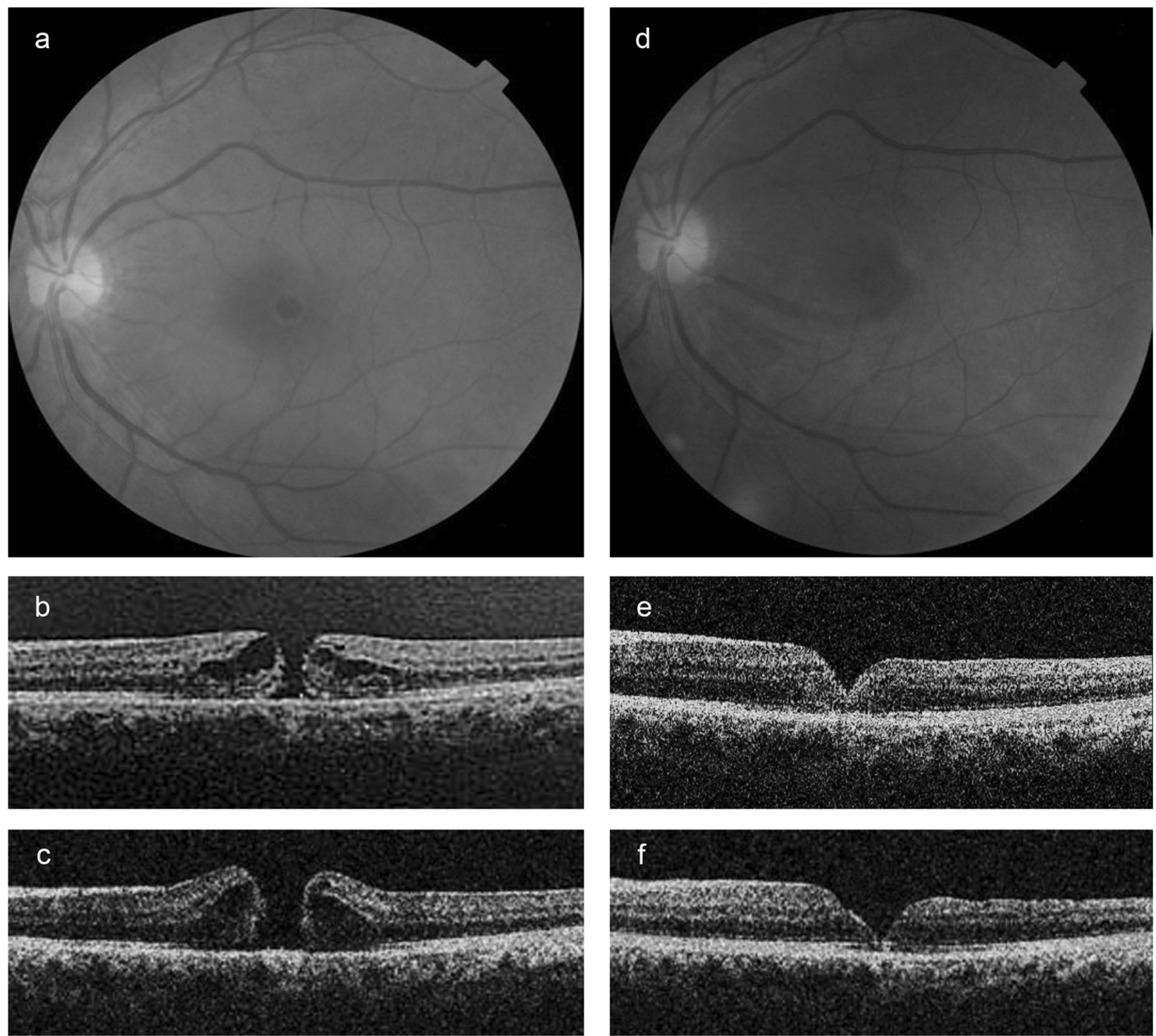

Fig. 3 Fundus photographs and optical coherence tomography (OCT) images of the small full-thickness macular hole (FTMH) in left eye with a diameter of $191 \mu \mathrm{m}$, without vitreomacular traction (VMT), before (a, b) and 1 month (c) after intravitreal injection of expansile gas. The hole progressed to a medium FTMH with a diameter of 383

$\mu \mathrm{m}$. After 1 month of intravitreal gas injection, the patient was treated by subsequent vitrectomy. An anatomical closure of the hole was demonstrated by fundus photograph and OCT images at 2 weeks (e) and 2 months $(\mathbf{d}, \mathbf{f})$. Corrected visual acuity was decreased from 0.3 (pre-treatment) to 0.1 (post-treatment)

showed definite efficacy of intravitreal expansile gas alone for primary FTMH. However, there was no significant difference between mean pre-operative and post-operative BCVA in small FTMH group. This result may be due to small FTMHs only causing slightly decreases of visual acuity, so there was no significant difference after surgery. In medium and large FTMHs, there was a significant difference between mean pre-treatment and post-treatment BCVA. The result suggested that pneumatic vitreolysis (PV, IVI of an expansile gas bubble) is a convenient and an effective method for FTMHs.

We found that successful closure of FTMHs occurs with significantly higher frequency in smaller holes $(P=0.024)$. The average hole diameter of the closure group was $307.8 \pm$ $122.8 \mu \mathrm{m}$ and of the non-closure group was $431.6 \pm 128.4$ $\mu \mathrm{m}$. VMT was delineated by OCT in ten eyes, and all of them $(100 \%)$ were released after injection of the gas. Within the ten eyes, hole closure rate was $90 \%$ (9 of 10). VMT condition was significantly different between the closure group and the non-closure group $(P=0.038)$. These data suggest that IVI of expansile gas alone may promote macular hole closure through releasing VMT. 
Table 1 Summary of the study subjects

Case Gender Age Eye Hole diameter IVTS type VMT LogMAR

$\overline{\text { Pre-operative Post-operative }}$

\begin{tabular}{|c|c|c|c|c|c|c|c|c|c|}
\hline 1 & $\mathrm{~F}$ & 63 & OS & 167 & Small & + & 0.70 & 0.60 & Closed \\
\hline 2 & $\mathrm{~F}$ & 63 & OS & 223 & Small & + & 0.70 & 0.20 & Closed \\
\hline 3 & $\mathrm{~F}$ & 64 & OS & 110 & Small & - & 0.50 & 0.20 & Closed \\
\hline 4 & $\mathrm{~F}$ & 62 & OS & 173 & Small & - & 1.00 & 0.20 & Closed \\
\hline 5 & M & 74 & OS & 189 & Small & - & 0.70 & 0.10 & Closed \\
\hline 6 & M & 35 & OD & 174 & Small & - & 0.30 & 0.10 & Closed \\
\hline 7 & $\mathrm{~F}$ & 70 & OS & 270 & Medium & + & 1.00 & 0.20 & Closed \\
\hline 8 & $\mathrm{~F}$ & 77 & OD & 399 & Medium & + & 1.00 & 0.40 & Closed \\
\hline 9 & $\mathrm{~F}$ & 25 & OD & 255 & Medium & + & 0.40 & -0.10 & Closed \\
\hline 10 & M & 49 & OD & 327 & Medium & + & 0.60 & 0.20 & Closed \\
\hline 11 & M & 50 & OD & 327 & Medium & - & 1.40 & 0.20 & Closed \\
\hline 12 & $\mathrm{~F}$ & 37 & OD & 283 & Medium & - & 1.00 & 0.40 & Closed \\
\hline 13 & $\mathrm{~F}$ & 66 & OS & 384 & Medium & - & 0.60 & 0.20 & Closed \\
\hline 14 & $\mathrm{~F}$ & 60 & OD & 400 & Medium & - & 1.00 & 0.20 & Closed \\
\hline 15 & $\mathrm{~F}$ & 64 & OS & 454 & Large & + & 0.80 & 0.50 & Closed \\
\hline 16 & $\mathrm{~F}$ & 68 & OS & 542 & Large & + & 1.00 & 0.40 & Closed \\
\hline 17 & $\mathrm{~F}$ & 63 & OD & 495 & Large & + & 1.00 & 0.90 & Closed \\
\hline 18 & $\mathrm{~F}$ & 59 & OS & 191 & Small & - & 0.50 & 1.00 & Open \\
\hline 19 & $\mathrm{~F}$ & 60 & OD & 271 & Medium & - & 0.90 & 1.00 & Open \\
\hline 20 & $\mathrm{~F}$ & 62 & OS & 383 & Medium & - & 1.00 & 0.80 & Open \\
\hline 21 & $\mathrm{~F}$ & 67 & OS & 536 & Large & - & 1.50 & 0.80 & Open \\
\hline 22 & $\mathrm{~F}$ & 62 & OS & 567 & Large & - & 0.80 & 0.90 & Open \\
\hline 23 & $\mathrm{~F}$ & 60 & OD & 431 & Large & - & 1.00 & 1.00 & Open \\
\hline 24 & $\mathrm{~F}$ & 60 & OS & 533 & Large & - & 0.60 & 0.70 & Open \\
\hline 25 & $\mathrm{~F}$ & 71 & OD & 471 & Large & - & 1.30 & 1.15 & Open \\
\hline 26 & M & 64 & OD & 501 & Large & + & 1.00 & 0.60 & Open \\
\hline
\end{tabular}

$F$ female, $M$ male, VMT vitreomacular traction, $\log M A R$ logarithm of the minimum angle of resolution, + present, - absent

Following the surgery of a macular hole, a head-down positioning may be advised. According to previous study, Chan et al. [6] have reported the use of 0.3 to $0.5 \mathrm{ml}$ of $100 \% \mathrm{C} 3 \mathrm{~F} 8$, with the majority of patients positioning for 4-6 weeks. The closure rate for stage 2 macular hole in Chan's study was 50\% (3/6). Jorge et al. [7] have reported use of $0.4 \mathrm{ml}$ of $100 \% \mathrm{C}_{3} \mathrm{~F}_{8}$ and asked patients to maintain a head-down position of 1 week. They also got a $50 \%$ closure rate (3/6). We used $100 \% \mathrm{C}_{3} \mathrm{~F}_{8}$ of $0.2 \mathrm{ml}$ and asked the patients to maintain a prone position for 7 days, and extended the duration to 2 weeks in unsuccessful cases. Our success rate was $65.4 \%(17 / 26)$ and higher than previous studies. The value of face-down positioning after surgery was still controversial [13, 14]. We chose 7-14 days, according to our previous clinical experiences that gas bubble is almost absorbed 2 weeks after surgery. It is currently insufficient to draw firm conclusion and guide practice about the duration of face-down positioning after gas tamponade. More random controlled clinical trials are needed to give more evidences.
In this study of pneumatic treatment for FTMHs, there was complication of localized RD of one eye 2 months after the surgery. The patient who developed RRD had a myopia of $-5.0 \mathrm{DS}$ and 2 months after surgery was a time when the gas bubble had been absorbed. Another case with $-4.0 \mathrm{D}$ myopia developed RRD 10 months after the surgery. We thought that the reason why RRD developed was also related with myopia. But these two cases also reminded us that in eyes with idiopathic FTMH, there is partial vitreoretinal adhesion. Vitreo-foveal separation of partial PVD may occur after gas injection. Vitreous fibers may be stretched by gas expansion and cause retinal break, especially in areas of retinal degeneration [15]. To reduce this complication, peripheral retinal examination is essential, especially for the cases with myopia. The presence of lattice degeneration should be considered as a contraindication for pneumatic treatment of macular hole.

Some important weaknesses to our case series include low number of patients and no control group. The amount of cases was low when we performed the subgroup analysis, 
Table 2 Functional outcomes after treatment

\begin{tabular}{llll}
\hline & $\begin{array}{l}\text { Pre-treatment mean } \\
\text { logMAR BCVA }\end{array}$ & $\begin{array}{l}\text { Post-treatment mean } \\
\text { logMAR BCVA }\end{array}$ & $P$-value \\
\hline Total cases (26/26) & $0.84 \pm 0.29$ & $0.49 \pm 0.36$ & $<0.01^{*}$ \\
Treated by gas alone (17/26) & $0.81 \pm 0.28$ & $0.29 \pm 0.23$ & $<0.01^{*}$ \\
Treated by gas and PPV (9/26) & $0.94 \pm 0.32$ & $0.88 \pm 0.17$ & 0.616 \\
Small (7/26) & $0.63 \pm 0.22$ & $0.34 \pm 0.34$ & 0.123 \\
Medium (10/26) & $0.88 \pm 0.29$ & $0.35 \pm 0.32$ & $0.002^{*}$ \\
Large (9/26) & $1.00 \pm 0.27$ & $0.77 \pm 0.24$ & $0.048^{*}$ \\
\hline
\end{tabular}

$P P V$ pars plana vitrectomy, $\log M A R$ logarithm of the minimum angle of resolution, $B C V A$ best-corrected visual acuity

* Significant

which may cause biases of the result. A randomized study with larger amount of patients and appropriate controls will be needed to evaluate the exact effect of pneumatic treatment in FTMHs.

A previous study found that the morphology of the FTMH was predictive of closure in patients treated with ocriplasmin. They defined "width factor" as the base diameter minus the minimum linear diameter and found that FTMH patients who treated with ocriplasmin with a width factor $<60 \mu \mathrm{m}$ have an improved probability of closure [16]. This was not assessed in this study, but it is possible that this and other measures of hole shape may be useful in predicting success with expansile gas.

In summary, our study found that IVI of expansile gas alone can lead to a PVD and may have merit in selected cases to treat idiopathic FTMH. For the holes with diameters less than $400 \mu \mathrm{m}$ and pre-treatment VMT existing, a pneumatic approach had a higher success rate. Advantages of this procedure, as compared with traditional surgical approaches, may include ease of operation and anesthesia, limited invasiveness and procedure time, cost-saving, and lower rate of severe complications. Current vitrectomy surgery with or without ILM peeling is needed for the patients with large FTMH or retinal degeneration. Pneumatic treatment should be studied further in a prospective fashion to better delineate the benefits and risks associated with this promising procedure.

\section{Summary}

\section{What was known before}

- In previous studies, IVIs of expansile gas are useful ways to treat early idiopathic macular holes.

\section{What this study adds}

- We evaluated the efficacy of IVI of expansile gas for primary FTMH (small/medium/large FTMHs), which were not showed in previous articles.
- We found that IVI of expansile gas alone can lead to a PVD and may have merit in selected cases to treat idiopathic FTMH.

\section{Compliance with ethical standards}

Conflict of interest The authors declare that they have no conflict of interest.

\section{References}

1. Gass JD. Idiopathic senile macular hole: its early stages and pathogenesis. Arch Ophthalmol. 1988;106:629-39.

2. Gass JD. Reappraisal of biomicroscopic classification of stages of development of a macular hole. Am J Ophthalmol. 1995;120:808-9.

3. Duker JS, Kaiser PK, Binder S, et al. The international vitreomacular traction study group classification of vitreomacular adhesion, traction, and macular hole. Ophthalmology. 2013;120:2611-9.

4. Banker AS, Freeman WR, Kim JW, et al. Vision-threatening complications of surgery for full-thickness macular holes. Vitrectomy for macular hole study group. Ophthalmology. 1997; 104:1442-52.

5. Park SS, Marcus DM, Duker JS, et al. Posterior segment complications after vitrectomy for macular hole. Ophthalmology. 1995;102:775-81.

6. Chan CK, Wessels IF, Friedrichsen EJ. Treatment of idiopathic macular holes by induced posterior vitreous detachment. Ophthalmology. 1995;102:757-67.

7. Jorge R, Costa RA, Cardillo JA, et al. Optical coherence tomography evaluation of idiopathic macular hole treatment by gasassisted posterior vitreous detachment. Am J Ophthalmol. 2006;142:869-71.

8. Mori K, Saito S, Gehlbach PL, et al. Treatment of stage 2 macular hole by intravitreous injection of expansile gas and induction of posterior vitreous dectachment. Ophthalmology. 2007;114:127-33.

9. Chen TC, Yang CH, Yang CM. Intravitreal expansile gas in the treatment of early macular hole: reappraisal. Ophthalmologica. 2012;228:159-66

10. Ip MS, Baker BJ, Duker JS, et al. Anatomical outcomes of surgery for idiopathic macular hole as determined by optical coherence tomography. Arch Ophthalmol. 2002;120:29-35.

11. Ullrich S, Haritoglou C, Gass C, et al. Macular hole size as a prognostic factor in macular hole surgery. $\mathrm{Br} \mathrm{J}$ Ophthalmol. 2002;86:390-3. 
12. Chang S. Controversies regarding internal limiting membrane peeling in idiopathic epiretinal membrane and macular hole. Retina. 2012;32:S200-3; discussion S203-4.

13. Solebo AL, Lange CAK, Bunce $C$, et al. Face-down positioning or posturing after macular hole surgery. Cochrane Database Syst Rev. 2011;12:Art. No.: CD008228.

14. Parravano M, Giansanti F, Eandi CM, et al. Vitrectomy for idiopathic macular hole. Cochrane Database Syst Rev. 2015;5: Art. No.: CD009080.
15. Cullinane AB, Cleary PE. Prevention of visual field defects after macular hole surgery. $\mathrm{Br} \mathrm{J}$ Ophthalmol. 2000;84:372-7.

16. Steel DH, Parkes C, Papastavrou VT, et al. Macular hole closure with ocriplasmin based on spectral domain optical coherence tomography. Eye. 2016;30:740-5. 